\title{
2.4/5.7-GHz Dual-Band Dual-Conversion Architecture With Correlated $L O$ Signal Generators
}

\author{
Jin-Siang Syu ${ }^{1}$, Chinchun Meng ${ }^{1}$, Sheng-Wen $\mathrm{Yu}^{1}$, Tzung-Han $\mathrm{Wu}^{1}$ and Guo-Wei Huang ${ }^{2,3}$ \\ ${ }^{1}$ Department of Communication Engineering, National Chiao Tung University, Hsinchu, 300 Taiwan, R.O.C. \\ ${ }^{2}$ Department of Electronics Engineering, National Chiao Tung University, Hsinchu, 300 Taiwan, R.O.C. \\ ${ }^{3}$ National Nano Device Laboratories, Hsinchu, 300 Taiwan, R.O.C.
}

\begin{abstract}
A 2.4/5.7-GHz dual-band dual-conversion downconverter is demonstrated using $0.35-\mu \mathrm{m}$ SiGe heterojunction bipolar transistor (HBT) technology. This downconverter employs a complex dual-conversion system and a complex filter to reject the first and second image signals, respectively. The $L O_{1}$ frequency is 2.5 times the $\mathrm{LO}_{2}$ frequency and is generated by a divide-by-two divider, a frequency doubler and a single-sideband up-converter. As a result, the correlated $L O$ signals maintain excellent image-rejection performance of the dual-conversion system while only one $L O$ input is needed. The downconverter achieves a 40/39-dB image-rejection ratio of the first image and a 44/46-dB image-rejection ratio of the second image for 2.4/5.7-GHz modes, respectively.
\end{abstract}

Index Terms - Wireless LAN, dual-band, SiGe heterojunction bipolar transistor (HBT), low-IF, image rejection.

\section{INTRODUCTION}

Compared with two (or multiple)-separate-system solutions for multi-band applications, the dual-band (or multi-band) reconfigurable system has a relatively high integration level. For dual-band transceivers, the direct-conversion architectures were demonstrated [1]-[4]. Besides, some dual-band dualconversion systems were demonstrated while the signal was down-converted to baseband $\left(I F_{2}=0\right)$ at the second stage; thus, there is no second image problem [5] but the flicker noise and dc offset problems arise and need more efforts to solve [6]-[7]. Most of these zero- $I F$ multi-band receivers require more than one local oscillator [1]-[3]. Since $I F_{2}=0$, it is difficult to use one VCO covering both bands. The extra VCO may cause substrate cross-talk and spurious signals.

In this paper, a $2.4 / 5.7-\mathrm{GHz}$ dual-band low- $I F$ system for WLAN $802.11 \mathrm{a} / \mathrm{g}$ applications is demonstrated. Since the low- $I F$ architecture keeps the $I F$ frequency flexible, the $L O_{1}$ and $\mathrm{LO}_{2}$ can be set as a fractional multiple of each other. Thus, the $L O_{1}$ frequency is 2.5 times the $L O_{2}$ frequency in this work and thus only one $L O$ signal source is needed. Besides, correlated $L O$ signals can maintain an excellent imagerejection performance as long as phase errors at the first and second $L O$ differential-quadrature signals are the same [8].

The dual-conversion low- $I F$ system of this work combines the Weaver architecture [8]-[10] and Hartley architecture [11]. The former is a complex dual-conversion topology that removes the image signal by a frequency-shifting mechanism while the latter is a complex down-conversion with a complex poly-phase filter to remove the image. In a complex mixer topology, the signal spectrum shifts in one way, either upwards or downwards, as long as the $L O$ signal is perfectly quadrature [9]. As a result, different polarities of the $L O$ signal result in different operating modes.

The demonstrated dual-band system performs excellent image-rejection performance because the double-quadrature system of the second stage has much immunity of an amplitude/phase error not only from a signal imbalance but also from device mismatches in the mixers.

\section{CIRCUIT DESIGN}

The block diagram of the $2.4 / 5.7-\mathrm{GHz}$ dual-band dualconversion downconverter is shown in Fig. 1. This circuitry consists of a single-quadrature system (two mixers) at the first stage, a double-quadrature system (four mixers) at the following stage and an $L O$ generator generating differentialquadrature signals of $L O_{1}$ and $L O_{2}$. An active Gilbert mixer is employed in both the first- and second-stage mixers for its advantages of a higher gain and a lower $L O$ power requirement. Besides, the first-stage mixer is a micromixer topology [12] consisting of common-emitter and commonbase configurations at the input stage; thus, the balanced output and wideband matching can be easily achieved.

The frequency multiplier with a multiple of 2.5 consists of a frequency doubler $(\times 2)$ and a frequency divider $(\div 2)$. After the mixing operation of a single-sideband (SSB) up-converter, the $L O_{1}\left(2.5 \times L O_{2}\right)$ signal is thus generated. Both differentialquadrature signals of the $L O_{1}$ and $L O_{2}$ are generated by a twosection poly-phase filter with the center frequency of 4.05 and $1.62 \mathrm{GHz}$, respectively. Figure 2(a) shows the block diagram of the static divider consisting of two D-latches realized by emitter-coupled logic. The schematic of the D-latch, consisting of the sample and hold stages, is shown in Fig. 2(b). Instead of using a simple cross-coupled pair, the commoncollector configuration is inserted into the positive feedback loop at the hold stage to achieve a wider output swing and a higher achievable operating frequency.

Figure 3(a) shows the function block of the multiplier while the schematic of the multiplier is shown in Fig. 3(b). If $f_{1} \neq f_{2}$, the multiplier is an SSB up-converter while the $\left(f_{1}-f_{2}\right)$ frequency component is cancelled if the two input signals have a perfect quadrature phase. On the other hand, if $f_{1}=f_{2}=f_{0}$, 


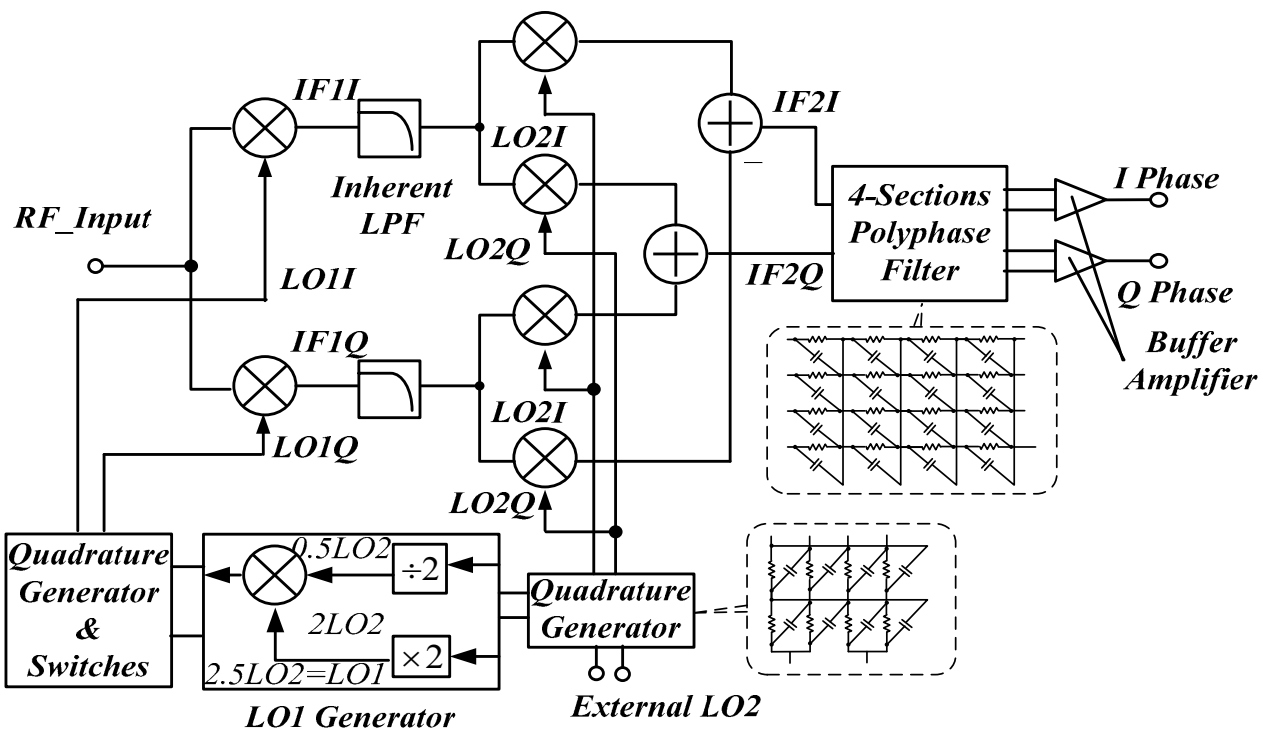

Fig. 1. Block diagram of the 2.4/5.7-GHz dual-band dual-conversion downconverter with correlated LO signal generators.

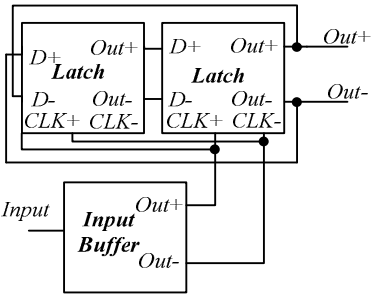

(a)



(b)
Fig. 2. (a) Block diagram of the static divide-by-two divider (2) the D-latch cell used in the divider.

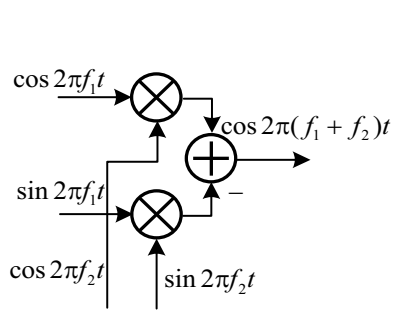

(a)

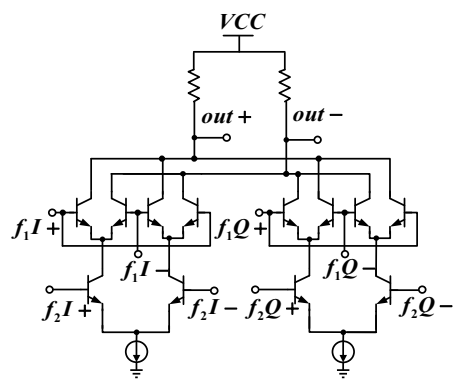

(b)
Fig. 3. (a) Function block of the multiplier which can be utilized as a single-sideband upconverter and a frequency doubler (b) schematic of the multiplier.

the circuit becomes a frequency doubler with the output frequency of $2 f_{0}$.

In the complex mixer topology of the first stage, the mixing operation leads to the frequency spectrum right-shifting, i.e., $f_{I F 1}=\left(-f_{R F}\right)+f_{L O 1}$, if the differential-quadrature $L O_{1}$ has the positive output sequence $\left(0^{\circ}, 90^{\circ}, 180^{\circ}\right.$ and $\left.270^{\circ}\right)$. On the other hand, if the polarity of the $L O_{1}$ is reversed, the output

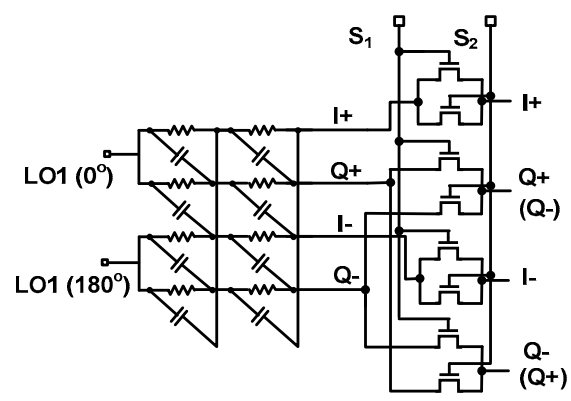

Fig. 4. Function block of the multiplier which can be utilized as a single-sideband upconverter and a frequency doubler (b) schematic of the multiplier.

spectrum is left-shifting, i.e., $f_{I F}=f_{R F}-f_{L O 1}$. By setting the $L O_{1}$ frequency at $4.05 \mathrm{GHz}$, halfway between $2.4 \mathrm{GHz}$ and 5.7 $\mathrm{GHz}$, the 2.4-GHz receiving mode employs a positive $L O_{1}$ sequence $\left(0^{\circ}, 90^{\circ}, 180^{\circ}\right.$ and $\left.270^{\circ}\right)$ while the $5.7-\mathrm{GHz}$ receiving mode employs a negative $L O_{1}$ sequence $\left(0^{\circ}, 270^{\circ}, 180^{\circ}\right.$ and $90^{\circ}$ ). As a result, the outputs at the first stage are both downconverted to $1.65 \mathrm{GHz}$ with positive $I / Q$ output sequence when the desired signal is received for both modes. Therefore, the dual-band operation can be achieved. The schematic of the switch of the $I / Q$ signal polarity is shown in Fig. 4. When $\left(\mathrm{S}_{1}, \mathrm{~S}_{2}\right)=(\mathrm{L}, \mathrm{H})$, the $5.7-\mathrm{GHz}$ band is selected. On the other hand, the 2.4-GHz band is chosen if $\left(\mathrm{S}_{1}, \mathrm{~S}_{2}\right)=(\mathrm{H}, \mathrm{L})$.

\section{MEASUREMENT RESULTS}

Figure 5 shows the microphoto of the 2.4/5.7-GHz dualband dual-conversion downconverter with correlated $L O$ signal generators and the die size is $1.63 \times 1.52 \mathrm{~mm}^{2}$. On-wafer measurement is employed for the $R F$ performance. The total power consumption is $198 \mathrm{~mW}$ at a $3.3-\mathrm{V}$ supply. Figure 6 shows the conversion gain (CG) and the single-sideband noise 


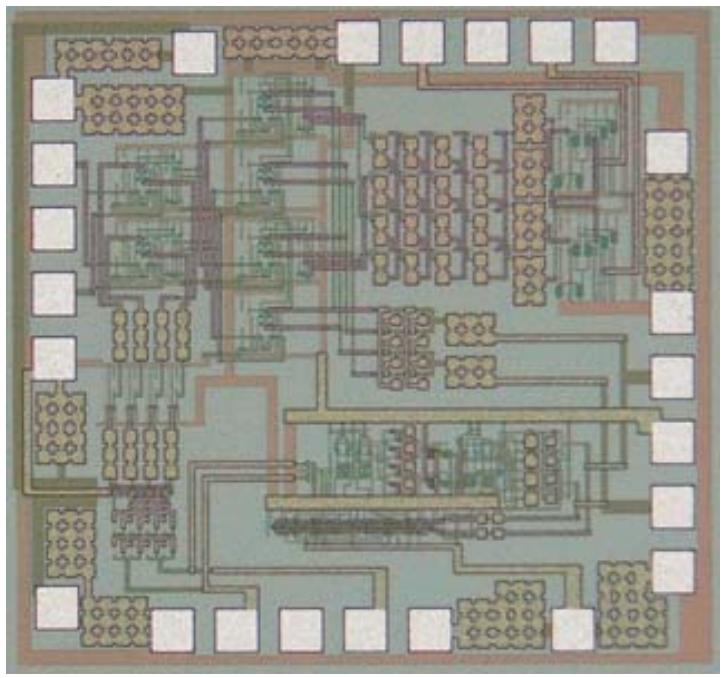

Fig. 5. The microphoto of the 2.4/5.7-GHz dual-band dualconversion downconverter with correlated LO signal generators.

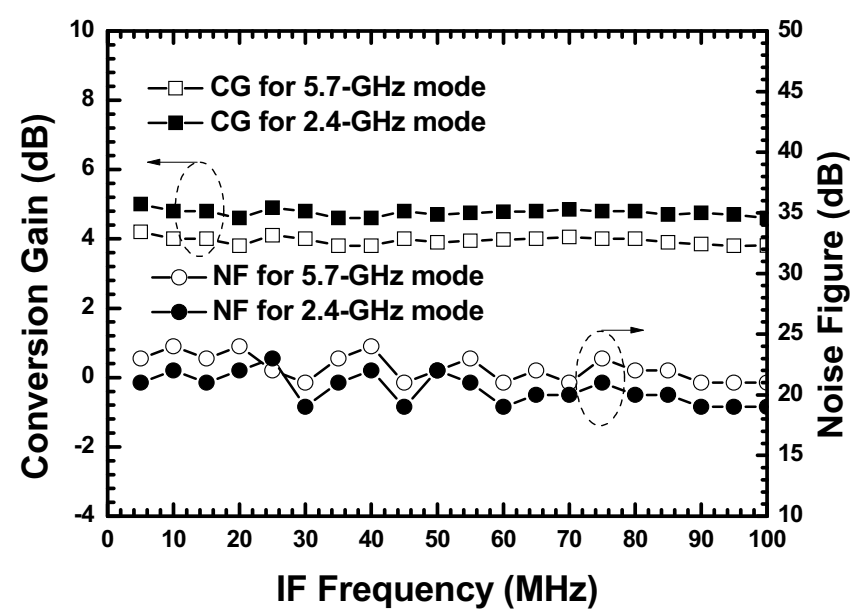

Fig. 6. Conversion gain and noise figure of the 2.4/5.7-GHz dualband dual-conversion downconverter with correlated LO signal generators.

figure (SSB-NF) of $2.4 / 5.7-\mathrm{GHz}$ bands The $\mathrm{CG}$ is $5 / 4 \mathrm{~dB}$ while the NF is about $20 \mathrm{~dB}$ for $2.4 / 5.7-\mathrm{GHz}$ band while the $I F$ frequency is below $100 \mathrm{MHz}$ and the $L O$ power is only 2 $\mathrm{dBm}$. Besides, the image-rejection ratios (IRRs) of both the first and second image signals for $2.4-\mathrm{GHz}$ band are shown in Fig. 7. The $I R R_{1}$ is above $40 \mathrm{~dB}$ and is flat due to the one-way frequency shifting. Compared with the $I R R_{1}$, the $I R R_{2}$ is $44-\mathrm{dB}$ within a narrow band from 15 to $45 \mathrm{MHz}$ due to the frequency response of the four-section poly-phase filter following the second-stage mixers. On the other hand, the $I R R_{1}$ and $I R R_{2}$ are 39 and $46 \mathrm{~dB}$ within the $I F$ bands from 15 to $45 \mathrm{MHz}$ for the 5.7- $\mathrm{GHz}$ mode as shown in Fig 8. Figure 9 shows the power performance of both $2.4 / 5.7-\mathrm{GHz}$ bands. The $I P_{1 \mathrm{~dB}}$ is $-12 /-9$ $\mathrm{dBm}$ while the $I I P_{3}$ is $2 / 6 \mathrm{dBm}$ for $2.4 / 5.7-\mathrm{GHz}$ band when $I F=30 \mathrm{MHz}$. The output waveform of both $I / Q$ channels are shown in Fig. 10 and the figure shows a $0.46 \mathrm{~dB}$ magnitude mismatch and a $0.62^{\circ}$ phase error.

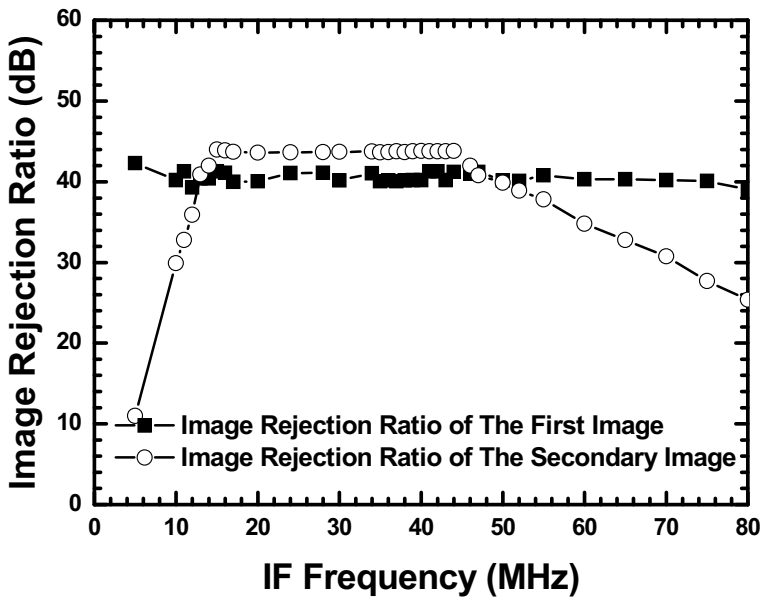

Fig. 7. Image rejection ratios of the first and second image signals at 2.4-GHz mode of the dual-band dual-conversion downconverter.

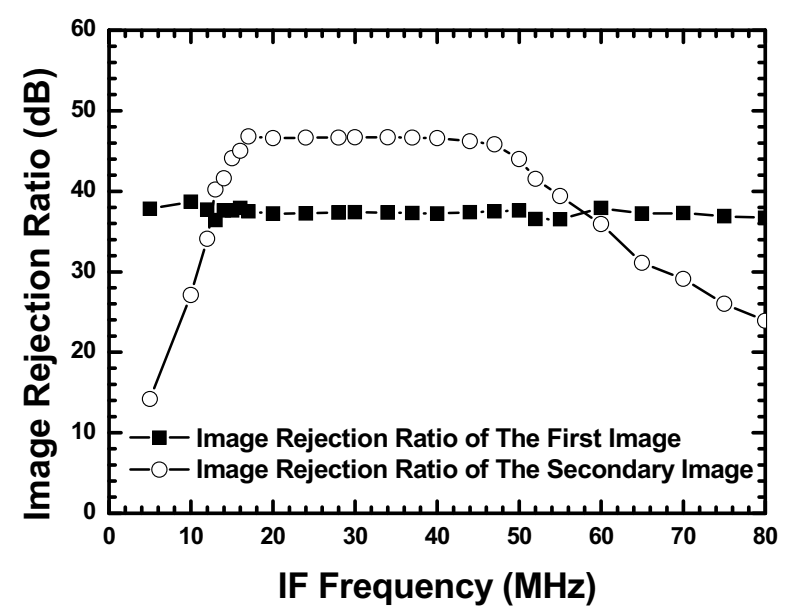

Fig. 8. Image rejection ratios of the first and second image signals at 5.7-GHz mode of the dual-band dual-conversion downconverter .

\section{CONCLUSION}

A highly-merged dual-band dual-conversion low- $I F$ downconverter for WLAN $802.11 \mathrm{a} / \mathrm{g}$ applications is demonstrated in this paper. The band selection is achieved by changing the polarity of the first $L O$ signals and thus $2.4-\mathrm{GHz}$ and 5.7-GHz bands are the image band of each other. Besides, only one signal generator is needed for the dual-conversion system. Since the two $L O$ signals are highly correlated, excellent $I R R$ s still maintain. As a result, $I R R_{1} / I R R_{2}$ for $2.4-$ $\mathrm{GHz}$ mode are $40 / 44 \mathrm{~dB}$ while a $39-\mathrm{dB} I R R_{1}$ and a $46-\mathrm{dB} I R R_{2}$ are achieved for the 5.7-GHz mode when $I F$ frequency ranging from 15 to $45 \mathrm{MHz}$.

\section{ACKNOWLEDGEMENT}

This work is supported by National Science Council of Taiwan, Republic of China under contract numbers NSC 952221-E-009-043-MY3, NSC 97-2221-E-009-171 and NSC 98- 


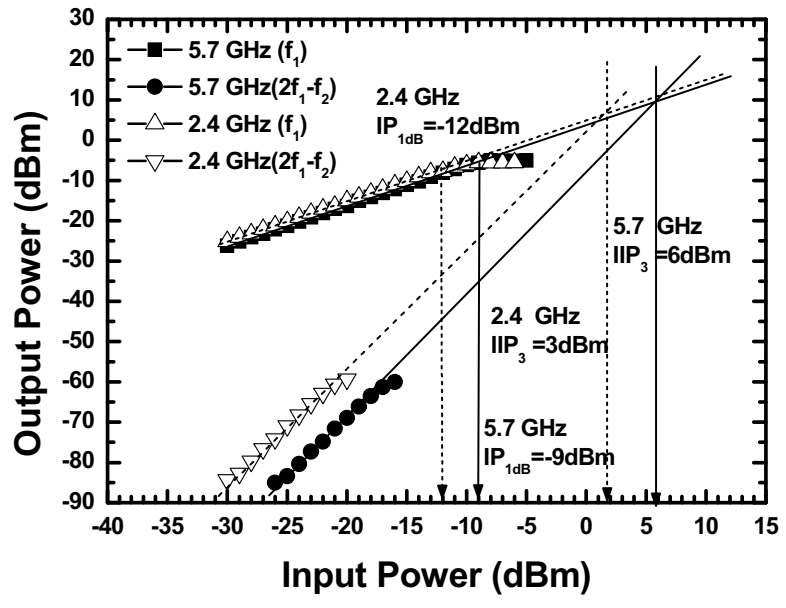

Fig. 9. Power performance of the 2.4/5.7-GHz dual-band dualconversion downconverter with correlated LO signal generators.

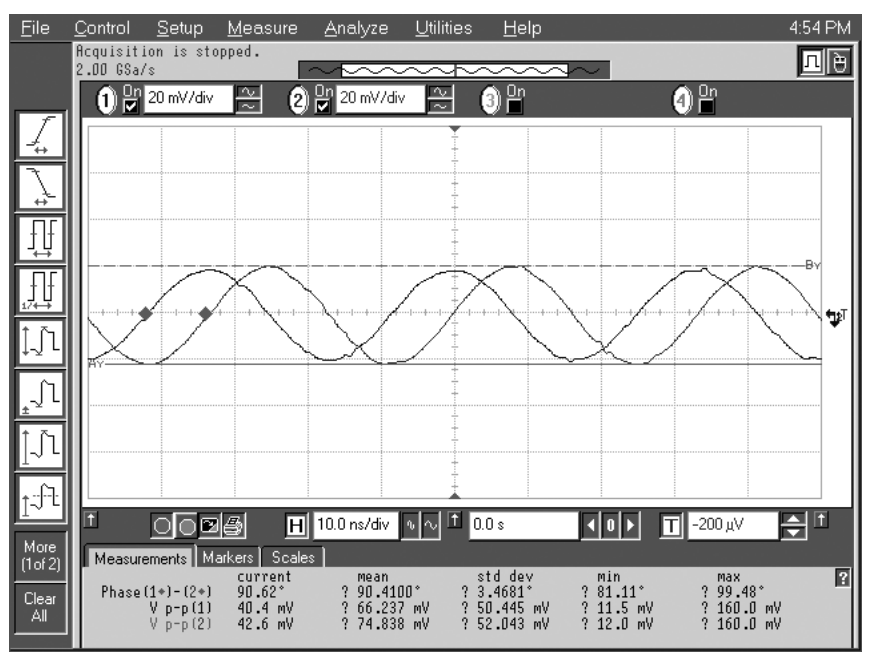

Fig. 10. Output I/Q waveforms of the $2.4 / 5.7-\mathrm{GHz}$ dual-band dualconversion downconverter with correlated LO signal generators.

2218-E-009-008-MY3, by the Ministry of Economic Affairs of Taiwan under contract number 96-EC-17-A-05-S1-020, and by MoE ATU Program under contract number 95 W803. The authors would like to thank National Chip Implementation Center (CIC) for technical support.

\section{REFERENCES}

[1] R. Ahola, A. Aktas, J. Wilson, K. R. Rao, F. Jonsson, I. Hyyryläinen, A. Brolin, T. Hakala, A. Friman, T. Mäkiniemi, J. Hanze, M. Sandén, D. Wallner, Y. Guo, T. Lagerstam, L. Noguer, T. Knuuttila, P. Olofsson, and M. Ismail, "A single- chip CMOS transceiver for $802.11 \mathrm{a} / \mathrm{b} / \mathrm{g}$ wireless LANs," IEEE J. Solid-State Circuits, vol. 39, no. 12, pp.2250-2258, Dec. 2004.

[2] O. Charlon, M. Locher, H. A. Visser, D. Duperray, J. Chen, M. Judson, A. L. Landesman, C. Hritz, U. Kohlschuetter, Y. Zhang, C. Ramesh, A. Daanen, M. Gao, S. Haas, V. Maheshwari, A. Bury, G. Nitsche, A. Wrzyszcz, W. R. White, H. Bonakdar, R. E. Waffaoui, and M. Bracey, "A low-power high-performance $\mathrm{SiGe} \mathrm{BiCMOS} 802.11 \mathrm{a} / \mathrm{b} / \mathrm{g}$ transceiver IC for cellular and Bluetooth co-existence applications", IEEE J. Solid-State Circuits, vol. 41, no. 7, pp. 1503-1512, Jul. 2006.

[3] M. Zargari, M. Terrovitis, S. H. M. Jen, B. J. Kaczynski, M. Lee, M. P. Mack, S. S. Mehta, S. Mendis, K. Onodera, H. Samavati, W. W. Si, K. Singh, A. Tabatabaei, D. Weber, D. K. $\mathrm{Su}$, and B. A. Wooley, "A single-chip dual-band tri-mode CMOS transceiver for IEEE $802.11 \mathrm{a} / \mathrm{b} / \mathrm{g}$ wireless LAN", IEEE J. Solid-State Circuits, vol. 39, no. 12, pp. 2239-2249, Dec. 2004.

[4] A. Behzad, K. A. Carter, H. -M. Chien, S. Wu, M.-A. Pan, C. P. Lee, Q. Li, J. C. Leete, S. Au, M. S. Kappes, Z. Zhou, D. Ojo, L. Zhang, A. Zolfaghari, J. Castanada, H. Darabi, B. Yeung, A. Rofougaran, M. Rofougaran, J. Trachewsky, T. Moorti, R. Gaikwad, A. Bagchi, J. S. Hammerschmidt, J. Pattin, J. J. Rael, and B. Marholev, "A fully integrated MIMO multiband direct conversion CMOS transceiver for WLAN applications (802.11n)", IEEE J. Solid-State Circuits, vol. 42, no. 12, pp. 2795-2808, Dec. 2007.

[5] S. Wu and B. Razavi, "A 900-MHz/1.8-GHz CMOS receiver for dual-band applications," IEEE J. Solid-State Circuits, vol. 33, no.12, pp. 2178-2185, Dec. 1998.

[6] A. A. Abidi, "Direct-conversion radio transceivers for digital communications," IEEE J. Solid-State Circuits, vol. 30, no. 12, pp.1399-1410, Dec. 1995.

[7] H. Darabi, and A. A. Abidi, "Noise in RF-CMOS mixers: a simple physical model," IEEE J. Solid-State Circuits, vol. 35, no. 1 , pp. 15-25, Jan. 2000.

[8] T. H. Wu, and C. C. Meng, "5.2/5.7GHz 48dB Image Rejection GaInP/GaAs HBT Weaver Down-Converter Using LO Frequency Quadrupler," IEEE J. Solid-State Circuits, vol. 41, no. 11, pp.2468-2480, Nov. 2006.

[9] D. Weaver, "A third method of generation and detection of single-sideband signals," Proceedings of The IRE, pp. 17031705, Dec. 1956.

[10] J. C. Rudell, J.-J. Ou, T. B. Cho, G. Chien, F. Brianti, J. A. Weldon, and P. R. Gray, "A 1.9-GHz wide-band IF double conversion CMOS integrated receiver for cordless telephone applications," IEEE J. Solid-State Circuits, vol. 32, no.12, pp. 2071-1088, Dec. 1997.

[11] F. Behbahani, Y. Kishigami, J. Leete, and A. A. Abidi, "CMOS mixers and polyphase filters for large image rejection," IEEE J. Solid-State Circuits, vol. 36, no.6, pp. 873-887, Jun. 2001.

[12] B. Gilbert, "The MICROMIXER: A highly linear variant of the Gilbert mixer using a bisymmetric Class-AB input stage," IEEE J. Solid-State Circuits, vol. 32, pp. 1412-1423, Sept. 1997. 